\title{
Control of animal products contamination with nitroimidazole group antibiotics
}

\author{
Alexandr Prosekov, Tatiana Podlegaeva*, Olga Chaplygina, Oksana Kozlova, and Anna \\ Tulaeva \\ ${ }^{1}$ Kemerovo State University, 650000, Krasnaya str., 6, Kemerovo, Russia
}

\begin{abstract}
Antibiotic contamination of water and food is a particularly important environmental worldwide problem. Antibiotics are found in water, soil, and food, which negatively affects both the environment and the health of the entire world's population. Therefore, it is important to control the content of antimicrobials. To solve this problem, scientists are developing new and improving existing methods for these preparation determination. The paper considers the possibility of applying the technique of high-performance liquid chromatography with mass spectrometric detection for the determination of nitroimidazole group antibiotics in meat raw materials. The method was evaluated in terms of the main characteristics taken into account during validation: accuracy, reproducibility, linearity, and specificity. Studies conducted on pork muscle tissue samples showed the absence of antibiotics of this group in all samples, which meets the requirements of regulatory documents.
\end{abstract}

\section{Introduction}

Antibiotics are firmly established in our lives primarily as drugs for therapeutic purposes for animals and humans. And as experts note, many diseases can be overcome only with the help of antibiotics. However, in modern animal husbandry, drugs of this group are often used for the prevention of diseases, and as stimulating drugs that allow to increase the muscle mass of animals and poultry in a short time. Antibiotic residues end up in meat, milk, and other animal products. And since it is not easy to remove antibiotics by simple methods, these drugs enter the human body, forming resistant strains of microorganisms, hypersensitivity of the body, intestinal microbiota dysbiosis. This problem causes serious concerns around the world $[1,2,3]$.

Monitoring farm animals for antibiotic residues is one of the main principles of the European Union (EU) and national monitoring programs. Technical regulation of the Customs Union TR CU 021/2011"On food safety" restricts or does not allow the content of veterinary drugs in raw materials. Commission decision 2002/657/EC specifies the investigation method as the method to be used to detect the presence of a substance or class of substances at a certain level. The method should be able to detect a specific metabolite or source compound at or below the appropriate regulatory limit.

\footnotetext{
${ }^{*}$ Corresponding author: tpodlegaeva@yandex.ru
} 
The world scientific community is developing simple and precise methods for control on various groups of antibiotics [4-7].

Nitroimidazoles - a group of drugs that are highly active against anaerobic bacteria, as well as pathogens of protozoal infections. Metronidazole - the first drug of the group was approved for medical use in 1960. Subsequently, other drugs were created that are still used to treat cattle, pigs and poultry for therapeutic or preventive purposes.

After the last use of the drug, animal slaughter is allowed no earlier than three days later, poultry - after 5 days. The use of milk from cows that have undergone antimicrobial therapy is allowed no earlier than 36 hours.

The use of food products containing a residual content of antimicrobial drugs, including nitroimidazole groups, in the diet can contribute to the emergence of addiction (resistance) to each group of drugs and the development of resistant forms of microorganisms. In addition, antibiotics can penetrate water and soil, which can lead to serious environmental consequences. Therefore, it is very important to control the contamination with antimicrobial drugs.

\section{Materials and methods}

The residual content of nitroimidazoles was determined by high-performance liquid chromatography with mass spectrometric detection (HPLC-MS/MS). Chromatomass spectrometer LCMS-8040 Shimadzu Corporatio with a cartridge for solid-phase extraction Strata C-18E $500 \mathrm{mg} / 6 \mathrm{~cm}^{3}$ or $500 \mathrm{mg} / 12 \mathrm{~cm}^{3}$ Phenomenex was used in work.

The study used reagents for HPLC: formic acid, 98\%, (CATROSA), Acetonitrile, (HPLC, sigma-aldrich), N-hexane, hc, TC 2631-158-44493179-13. The mobile phase used: mobile phase B - methanol. As the mobile phase A - $0.1 \%$ formic acid in deionized water. To prepare a mobile phase A eluent solution, deionized water was added to a $1000 \mathrm{~cm}^{3}$ volumetric flask, followed by $1 \mathrm{~cm}^{3}$ of formic acid, and the volume was adjusted to the mark with deionized water. Range of defined values $\mathrm{m} / \mathrm{z}(2-2000)$ a.m.u. The measurement range of nitroimidazoles residual content is from 1.0 to $1000.0 \mu \mathrm{g} / \mathrm{kg}$.

Analytical standards: Dimetridazole-Dz (DMZ), Ronidazole-Dz (RNZ), IpronidazoleDZ (IPZ), Hydroxypronidazole-Dz (IPZOH), Metronidazole-D4 (MNZ), Hydroxymethylmetronidazole-Dz (HMMNI), Ronidazole-Dz (RNZ).

Preparation of initial individual solutions of nitroimidazoles with a mass concentration of $200 \mu \mathrm{g} / \mathrm{cm}^{3}$ in methanol was carried out in centrifuge tubes with a capacity of $15 \mathrm{~cm}^{3}$. Then the solutions were placed for $1 \mathrm{~min}$ in an ultrasonic bath, kept at room temperature in a dark place for at least 20 minutes. Solutions storage period 3 months at a temperature from $0^{\circ} \mathrm{C}$ to $8^{\circ} \mathrm{C}$.

Preparation of individual solutions of nitroimidazoles with a mass concentration of 0.2 $\mu \mathrm{g} / \mathrm{cm}^{3}$ and $0.5 \mu \mathrm{g} / \mathrm{cm}^{3}$ in deionized water (for method formulation) was carried out in vials with a capacity of $2 \mathrm{~cm}^{3}$. As well as the initial solutions they were placed in an ultrasonic bath for $1 \mathrm{~min}$. Before further use, all solutions were kept at room temperature in a dark place for at least 20 minutes. Freshly prepared solutions were used without storage.

Preparation of solutions of nitroimidazoles mixture with mass concentration of 10 $\mu \mathrm{g} / \mathrm{cm}^{3}, 1 \mu \mathrm{g} / \mathrm{cm}^{3}$ and a mixture of internal standards of nitroimidazoles with mass concentration of $1 \mu \mathrm{g} / \mathrm{cm}^{3}$, used for preparation of the calibration solutions were performed in vials of dark glass with a capacity of $40 \mathrm{~cm}^{3}$. After preparation, the solution was placed in an ultrasonic bath for $1 \mathrm{~min}$. The solution was kept at room temperature in a dark place for at least 20 minutes. Solutions storage period 3 months at a temperature from $0^{\circ} \mathrm{C}$ to $8^{\circ} \mathrm{C}$.

Samples of pig meat were obtained from farms of the Kemerovo region. The muscle tissue of the meat samples was previously cleaned from coarse connective tissue, ground on a homogenizer and weighed on a scale $(\mathrm{m}=1.0 \mathrm{~g})$ in a polypropylene tube with a capacity of 
$15 \mathrm{~cm}^{3}$. A pipette dispenser was used to inject $0.04 \mathrm{~cm}^{3}$ of solution of internal nitroimidazoles standards mixture with a concentration of $0.4 \mu \mathrm{g} / \mathrm{cm}^{3}\left(400 \mathrm{ng} / \mathrm{cm}^{3}\right)$ each. The test tube was placed in a shaker for mixing for 15 minutes. Then $4 \mathrm{~cm}^{3}$ of acetonitrile was added and the test tube was placed in a shaker for extraction for 15 minutes. Then it was centrifuged at $4000 \mathrm{rpm}$ for 15 minutes at a temperature of $4{ }^{\circ} \mathrm{C}$. After centrifugation, the upper organic layer was poured into a polypropylene tube with a capacity of $15 \mathrm{~cm}^{3}$, placed on a heating module and evaporated in an air current at a temperature of $40{ }^{\circ} \mathrm{C}$ to $(0.1-0.2) \mathrm{cm}^{3} .2 \mathrm{~cm}^{3}$ of deionized water was added to the resulting residue, mixed and centrifuged at a speed of $4000 \mathrm{rpm}$ for 15 minutes at a temperature of $4{ }^{\circ} \mathrm{C}$.

Validation characteristics were evaluated by the following indicators: linearity, limit of detection (LOD), limit of quantification (LOQ), specificity, recovery (truth), accuracy (convergence, precision).

Linearity was determined by studying 5 concentration levels, each concentration level was determined at least 3 times. Then a calibration curve was made, and the concentrations of the calibration standards should cover the expected concentration range. The linearity criterion is the correlation coefficient.

The limit of detection (LOD) was determined by analyzing 10 samples of material enriched to the level corresponding to the criteria. The criteria for quantitative methods were determined experimentally with solutions with decreasing concentrations to the minimum values that meet the requirements for the method in accordance with 2002/657/EC.

The limit of quantification (LOQ) was determined by analyzing 10 samples of material enriched to the level corresponding to the criteria. The concentration defined as the limit of quantification (LOQ) was analyzed 10 times $(2002 / 657 / E C)$, the average deviation was calculated with the Grubbs criterion. The criterion for the limit of quantification (LOQ) is the signal-to-noise ratio.

Specificity was determined by examining 10 forms and 10 samples enriched at the level of the limit of quantification (LOQ). The specificity criterion for arbitration methods for enriched samples is the measurement result "detected" in accordance with SANCO/10684/2009.

Restoration (truth) was established using certified reference material. To do this, 6 repetitions of the material were studied in accordance with the testing instructions used for this method. Then the concentration of the analyte in each sample of repeats was determined. In conclusion, the average standard deviation and coefficient of variation for these concentrations were calculated.

The truth was determined as a percentage by dividing the average measured concentration by the certified value.

Convergence was determined by examining samples enriched with analyte at three different concentration levels: $1,1.5$, and 2 times the minimum operating limit or $0.5,1$, and 1.5 times the permitted limit; analyzing the samples and determining the concentration found in each sample; determining the average concentration, standard deviation, and variation coefficient (\%) of the enriched samples.

Quantitative determination of the nitroimidazoles residual content was performed using the internal standard method for the peak area of the identified compounds using a calibration curve. Detection of the analyzed samples was performed in the mode of selected reactions record.

To build calibration graphs, the LaBSolutions Browser data processing program, calculation methods created separately for each of the matrices were used. 


\section{Results and discussion}

The method of high-performance liquid chromatography is a sensitive method for determining residual antibiotics in the form and food products $[8,9,10]$. Therefore, it is often used in determining various drugs, including antibiotics.

However, several food products, including meat, are complex matrix, have a complex composition and structure, which makes it difficult to extract and detect antibiotics. Therefore, it is important to establish the methodology suitability for target use, demonstrate the adequacy (confirmation of compliance) of the implemented methodology.

The HPLC-MS/MS method was introduced as a sensitive method for determining antibiotics in many complex biological samples. MS / MS parameters such as gas flow rate, source temperature conditions, and collision gas have been adjusted to achieve maximum device response. In order to confirm the identity of the detected substances by quantitative methods, confirmatory criteria were established and described in Commission Decision 2002/657/EC 31. In addition, the change in the analyte retention time in an unknown sample and a standard working solution should not differ by more than $2.5 \%$.

The results of the experiment showed that the presented method is linear in the observed range $(1-1000) \mathrm{ng} / \mathrm{cm}^{3}$ with a high correlation coefficient $\left(\mathrm{R}^{2}\right)$ for all nitroimidazoles greater than 0.999 (table 1).

Table 1. Range of linearity.

\begin{tabular}{|c|c|c|c|c|}
\hline Matrix & $\begin{array}{c}\text { Nitroimidazol } \\
\mathrm{e}\end{array}$ & $\begin{array}{l}\text { Linearity } \\
\text { equation }\end{array}$ & $\begin{array}{c}\text { Correlation } \\
\text { coefficient }\end{array}$ & $\begin{array}{c}\text { Observed } \\
\text { linearity } \\
\text { range, } \mathrm{ng} / \mathrm{cm}^{3} \\
\end{array}$ \\
\hline \multirow{9}{*}{$\begin{array}{l}\text { Pork meat } \\
\text { (muscle } \\
\text { tissue) }\end{array}$} & DMZ & $0.189227 * X+0$ & 0.9998276 & $1-1000$ \\
\hline & RNZ & $0.464170 * \mathrm{X}+0$ & 0.9998107 & $1-1000$ \\
\hline & IPZ & $0.107758 * X+0$ & 0.9999664 & $1-1000$ \\
\hline & IPZOH & $0.955916 * X+0$ & 0.9997526 & $1-1000$ \\
\hline & MNZ & $1.47531 * X+0$ & 0.9997620 & $1-1000$ \\
\hline & MNZOH & $0.572021 * \mathrm{X}+0$ & 0.9999209 & $1-1000$ \\
\hline & HMMNI & $0.577487 * \mathrm{X}+0$ & 0.9997761 & $1-1000$ \\
\hline & TNZ & $1.62393 * X+0$ & 0.9997689 & $1-1000$ \\
\hline & TIN & $0.00722141 * X+0$ & 0.9998423 & $1-1000$ \\
\hline
\end{tabular}

When determining the limit of detection, no deviations were detected, and all enriched samples were determined. In this case, the acceptable probability of error ( $\beta$-error) was no more than $5 \%$ at $n \geq 20$.

In the specificity test, no interventions were recorded during retention of each of the target analytes.

Accuracy assessment and studies to determine convergence/repeatability and intralaboratory convergence were performed at three levels of concentration of each analyte: 50,100 and $150 \mu \mathrm{g} / \mathrm{kg}$. For each concentration, three determinations were carried out under the conditions of two changing factors: time, executor. The study was conducted by two executors. Each determination included six measurement results obtained under repeatability conditions. All measurement results were checked for emissions according to the Grubbs criteria. The average values of recovery indicators and some statistical indicators are presented in table 2 . 
Table 2. Average indicators of method validation characteristics.

\begin{tabular}{|c|c|c|c|c|c|}
\hline \multirow[t]{2}{*}{$\begin{array}{l}\text { Matrix/nitroimid } \\
\text { azole }\end{array}$} & \multirow[t]{2}{*}{$\begin{array}{l}\text { Recovery, } \% \\
\text { (average value) }\end{array}$} & \multirow{2}{*}{$\begin{array}{l}\text { Grubbs } \\
\text { criterion (G), } \\
\text { (no more than } \\
2.651)\end{array}$} & \multicolumn{3}{|c|}{ 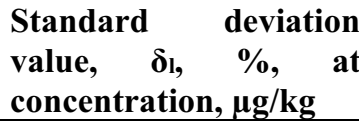 } \\
\hline & & & 50 & 100 & 150 \\
\hline DMZ & $72.2-86.6$ & $0.720-2.494$ & 18.6 & 14.3 & 10.9 \\
\hline RNZ & $75.5-101.0$ & $0.850-2.414$ & 8.8 & 8.7 & 7.6 \\
\hline IPZ & $89.1-116.2$ & $1.043-2.055$ & 10.2 & 7.2 & 5.4 \\
\hline IPZOH & $70.9-84.5$ & $1.012-1.934$ & 10.6 & 15.1 & 9.1 \\
\hline MNZ & $69.3-91.8$ & $0.723-2.462$ & 12.4 & 17.9 & 11.2 \\
\hline HMMNI & 70.7-109.9 & $0.918-2.418$ & 8.9 & 11.4 & 8.6 \\
\hline TNZ & $72.2-101.0$ & $0.882-1.846$ & 11.9 & 9.3 & 9.6 \\
\hline TIN & $94.6-117.1$ & $0.904-2.296$ & 10.1 & 7.3 & 8.1 \\
\hline
\end{tabular}

When determining the limit value of variation coefficients using the Hurwitz formula, the convergence variation coefficient was $30.3 \%$, and the reproducibility variation coefficient was $45.3 \%$. The Grubbs criterion in all cases did not exceed the calculated value of 2.651. The convergence evaluation also showed a high percentage.

LOD and LOQ at which the method criteria were met (average extraction in the range from 72 to $117 \%$ with $\mathrm{RSD} \leq 30 \%$ ). The nitroinidazoles extraction at all levels of enrichment was in the range from 75.6 to $115 \%$, with an RSD of less than $15 \%$ under accuracy conditions and below $20 \%$ for intermediate accuracy, which indicates good accuracy and precision of the proposed method.

The method under these conditions was used to determine this group of antibiotics in the muscle tissue of pigs obtained from farms of the Kemerovo region. 7 samples were analyzed. According to the Decision of the Eurasian Commission No. 28 dated 13.02.2018, the content of nitroimidazole antibiotics is not allowed in products of animal origin. According to the conducted research, antibiotics of nitroimidazole group were not detected in all samples.

\section{Conclusions}

Thus, we evaluated the antibiotics degree of products of animal origin with nitroimidazole group antibiotics by high-performance liquid chromatography with mass spectrometric detection. In the course of the work, the main validation characteristics of this method for determining these drugs in meat raw materials were obtained. The results showed high accuracy, convergence, and linearity of the method, which makes it possible to use it for the study of complex matrices.

\section{Acknowledgment}

This work was supported by a grant from the President of the Russian Federation with state support of leading scientific schools (NSh-2694.2020.4)

\section{References}

1. G. Pozza., A.Pinto, S. Crovato et al. Italian J of animal science , 19(1), 905 (2020)

2. J.Griboff, J.Carrizo, uan Cruz; R.Bonansea et al, Food chem, 332, 127380 (2020) 
3. Y.Changwon, G.Song, W.Lim, Comparative biochemistry and physiology c-toxicology \& pharmacology237, 10884 (2020)

4. V. Gaudin, A. Rault, C. Hedou, C. Soumet, E. Verdon, Food addit contam Part A. 34, 510 (2017)

5. F. Granados-Chinchilla, C. Rodríguez, J Anal Methods Chem, 2017, 1315497 (2017)

6. JR Sophila, GD Raj, K. Kumanan, GS Chandra, S Vairamuthu, Pharma Innov J., 7, 753 (2018)

7. I.Kozarova, D.Juscakova, J.Simkova, et al., Italian J of animal science, 19 (1), 4 (2020)

8. O. Chaplygina, T. Podlegaeva, L. Ostroumov, Yu. Golubtsova, XXI century: results of the past and problems of the present plus, 3(51), 116 (2020)

9. A. Lugovskoy, D. Bolshakov, T. Nikeshina, Veterinary medicine today, 1(16), 30 (2016)

10. V. Amelin, A. Korotkov, Journal of analytical chemistry, 70(11), 1201 (2015)

11. A.Gajda, M.Antczak, K.Mitrowska and et.al. J.of separation science, 41(20), 3821 (2018)

12. D.Shin, H.Kang, J.Jeong and et al. F. analytical methods, 11(6), 1815 (2018) 